\title{
Cost-Effectiveness of Virtual Reality Cognitive Behavioral Therapy for Psychosis: Health-Economic Evaluation Within a Randomized Controlled Trial
}

Roos Pot-Kolder ${ }^{1}$, MSc; Wim Veling ${ }^{2}$, MD, PhD; Chris Geraets $^{2}$, MSc; Joran Lokkerbol ${ }^{3,4}, \mathrm{PhD}$; Filip Smit ${ }^{1,3,5}, \mathrm{PhD}$; Alyssa Jongeneel ${ }^{1,6}$, MSc; Helga Ising ${ }^{6}, \mathrm{PhD}$; Mark van der Gaag ${ }^{1,6}, \mathrm{PhD}$

\footnotetext{
${ }^{1}$ Department of Clinical, Neuro and Developmental Psychology, Vrije Universiteit, Amsterdam, Netherlands

${ }^{2}$ Department of Psychiatry, University Medical Centre Groningen, University of Groningen, Groningen, Netherlands

${ }^{3}$ Centre of Economic Evaluation and Machine Learning, Trimbos Institute, Utrecht, Netherlands

${ }^{4}$ Department of Health Care Policy, Harvard Medical School, Boston, MA, United States

${ }^{5}$ Department of Epidemiology and Biostatistics, University Medical Centers Amsterdam, Amsterdam, Netherlands

${ }^{6}$ Department of Psychosis Research, Parnassia Psychiatric Institute, The Hague, Netherlands
}

\section{Corresponding Author:}

Roos Pot-Kolder, MSc

Department of Clinical, Neuro and Developmental Psychology

Vrije Universiteit

Van der Boechorststraat 7

Amsterdam, 1081 BT

Netherlands

Phone: 31641034764

Email: roos.potkolder@gmail.com

\section{Abstract}

Background: Evidence was found for the effectiveness of virtual reality-based cognitive behavioral therapy (VR-CBT) for treating paranoia in psychosis, but health-economic evaluations are lacking.

Objective: This study aimed to determine the short-term cost-effectiveness of VR-CBT.

Methods: The health-economic evaluation was embedded in a randomized controlled trial evaluating VR-CBT in 116 patients with a psychotic disorder suffering from paranoid ideation. The control group $(n=58)$ received treatment as usual (TAU) for psychotic disorders in accordance with the clinical guidelines. The experimental group $(\mathrm{n}=58)$ received TAU complemented with add-on VR-CBT to reduce paranoid ideation and social avoidance. Data were collected at baseline and at 3 and 6 months postbaseline. Treatment response was defined as a pre-post improvement of symptoms of at least $20 \%$ in social participation measures. Change in quality-adjusted life years (QALYs) was estimated by using Sanderson et al's conversion factor to map a change in the standardized mean difference of Green's Paranoid Thoughts Scale score on a corresponding change in utility. The incremental cost-effectiveness ratios were calculated using 5000 bootstraps of seemingly unrelated regression equations of costs and effects. The cost-effectiveness acceptability curves were graphed for the costs per treatment responder gained and per QALY gained.

Results: The average mean incremental costs for a treatment responder on social participation ranged between $€ 8079$ and $€ 19,525$, with 90.74\%-99.74\% showing improvement. The average incremental cost per QALY was €48,868 over the 6 months of follow-up, with $99.98 \%$ showing improved QALYs. Sensitivity analyses show costs to be lower when relevant baseline differences were included in the analysis. Average costs per treatment responder now ranged between $€ 6800$ and $€ 16,597$, while the average cost per QALY gained was $€ 42,030$.

Conclusions: This study demonstrates that offering VR-CBT to patients with paranoid delusions is an economically viable approach toward improving patients' health in a cost-effective manner. Long-term effects need further research.

Trial Registration: International Standard Randomised Controlled Trial Number (ISRCTN) 12929657;
http://www.isrctn.com/ISRCTN12929657 


\section{KEYWORDS}

psychosis; virtual reality; cognitive behavioral therapy; cost-effectiveness

\section{Introduction}

Psychotic disorders impose a large disease burden-morbidity plus mortality - on the population, and in its wake, substantial economic costs occur for society and health care systems. The main drivers of societal costs of schizophrenia are health care costs and productivity losses, but patients and their families also incur substantial costs [1]. Low participation rates of individuals with psychosis in the labor market are an important cause of productivity losses, while the main contributor to health care costs are in-patient psychiatric admissions [2]. All in all, treatment costs of psychotic disorders consume a significant part of health care budgets in European countries [3].

Paranoid ideation is a common delusion in individuals with a psychotic disorder. Even when medicinal treatment is successful, paranoid thoughts and anxiety often remain because of conditioned avoidance and other acquired safety behaviors in social situations [4]. Social avoidance hinders recovery in social participation for patients and keeps unemployment rates as high as $70 \%-85 \%[5,6]$. A poor social network contributes to stigma and a lack of empowerment, resulting in more depressive symptoms and lower quality of life [7]. A smaller social network size is associated with more severe overall psychiatric and negative symptoms [8]. Virtual reality-based cognitive behavioral therapy (VR-CBT) was found to be an effective treatment for paranoid ideation in individuals with a psychotic disorder $[9,10]$. The use of virtual reality (VR) treatment in clinical practice is expected to become more widespread as VR technology becomes more readily available [11]. Therefore, information on the cost-effectiveness of this kind of treatment is required. This study was designed to evaluate whether adding VR-CBT to treatment as usual (TAU) would be effective in treating paranoid ideation in a cost-effective way with respect to improving social participation. A trial-based cost-effectiveness analysis (CEA) was conducted using data collected in seven outpatient mental health care services in the Netherlands, comparing add-on VR-CBT with TAU alone. This paper aims to determine the short-term (ie, 6-month) cost-effectiveness of VR-CBT from a societal perspective.

\section{Methods}

\section{Research Design}

The health-economic evaluation was embedded in a randomized controlled trial evaluating VR-CBT in 116 patients with a psychotic disorder suffering from paranoid ideation [10]. The VR-CBT study was a randomized, controlled, single-blind multicenter trial in two parallel groups, comparing add-on VR-CBT to TAU alone over a period of 6 months [10]. This study was approved by the Vrije Universiteit (VU University) Medical Ethics Committee for mental health service research and was registered retrospectively at the ISRCTN (International Standard Randomised Controlled Trial Number) registry (ISRCTN12929657). The trial protocol is provided elsewhere [12]. Four virtual social environments-a street, bus, café, and supermarket-were created with Vizard software (WorldViz). Within the environment, participants could move by operating a Logitech F310 Gamepad. They used a Sony HMZ-T1/T2/T3 head-mounted display with a high-definition resolution of 1280 $\times 720$ per eye, a 51.6 diagonal field of view, and a 3DOF (3 degrees of freedom) tracker for head rotation. VR-CBT therapists were psychologists with at least basic cognitive behavioral therapy (CBT) training. They received 2 days of training in VR-CBT. The VR-CBT manual described a structured treatment plan for all 16 sessions. Therapists were supervised in a group for 4 hours every month by two VR-CBT specialists.

\section{Recruitment}

Participants were recruited at seven treatment centers in the Netherlands between April 1, 2014, and December 31, 2015. To be included, participants had to meet the following criteria: (1) 18-65 years of age; (2) DSM-IV (Diagnostic and Statistical Manual of Mental Disorders, Fourth Edition) diagnosis of schizophrenia, schizophreniform disorder, schizoaffective disorder, delusional disorder, or psychotic disorder not otherwise specified; (3) suffering from at least mild paranoia, as assessed by Green's Paranoid Thoughts Scale (GPTS) (score of >40); and (4) self-report of avoiding at least one social situation. Exclusion criteria were as follows: (1) insufficient competency of Dutch language; (2) IQ below 70; and (3) a concurrent diagnosis of epilepsy. Assessments were performed at baseline and at 3 and 6 months postbaseline.

\section{Interventions}

All participants continued to receive TAU (ie, antipsychotic medication, regular contact with a psychiatrist to manage symptoms, and regular contact with a psychiatric nurse). Participants in the experimental condition also received therapist-led VR-CBT. VR-CBT treatment consisted of 16 biweekly sessions of 60 minutes each, using 40 minutes for exposure and behavioral exercises in virtual social environments. The therapist used an individual case formulation to help patients falsify their harm expectancies. No homework exercises were given between VR-CBT sessions. The treatment protocol, in Dutch, is available from the corresponding author.

\section{Outcome Measures}

\section{Overview}

We conducted both a CEA with three measures of improved social participation as outcome and a cost-utility analysis (CUA) with quality-adjusted life years (QALYs) gained as outcome. The outcome measures are described in more detail below.

\section{Social Participation}

The outcome of interest in the CEA was social participation. Social participation was operationalized in three ways: (1) objective social participation as the amount of time spent with others, (2) subjective social participation as momentary anxiety, and (3) subjective social participation as momentary paranoia. Momentary in this context meant that it was measured in real 
life during social company. All three outcomes were assessed in real time using the ecological sampling method (ESM). ESM is a structured diary method in which individuals are asked in daily life to report their thoughts, feelings, and symptoms, as well as the appraisal of the present social context. To that end, all participants carried an electronic device (PsyMate) for the ESM assessments. The device beeped at semirandom moments 10 times a day over 6 days. At each beep, the device collected self-assessments on a 7-point Likert scale ranging from 1 (not at all) to 7 (very). A positive treatment response on each of the three outcome measures was defined as an improvement of at least $20 \%$ at 6 months follow-up relative to the patient's baseline score.

\section{Quality-Adjusted Life Year}

The outcome in the CUA was the QALY derived from the GPTS [13]. The GPTS is an established broad measure of paranoid-delusional functioning that has long been used as an outcome measure. This instrument was chosen to be able to compare results with earlier CUA research on the subject. Mean GPTS scores at each measurement were first converted into the standard mean difference (SMD) by dividing the raw mean change scores by the SD of the GPTS at baseline in the control condition. In a next step, we used Sanderson et al's conversion factor [14] of 0.1835 (ie, the average of 0.209 using a rating scale and 0.158 using time trade-off), such that a change of 1 standard unit (ie, SMD) on the GPTS is equal to a corresponding change of 0.18 utility. The utility is a quality of life valuation and is needed to compute QALY gains in the VR-CBT condition relative to the TAU condition over the full 6 months between baseline and follow-up.

\section{Resource Use and Costing}

Societal costs were computed by adding (1) the direct medical costs of health care services use including the costs of antipsychotic medication and, in the experimental condition, the additional costs of adjunctive VR-CBT treatment; (2) direct nonmedical costs of travel; and (3) indirect costs stemming from lower productivity. For each participant, cost data over the last 3 months were collected at each of three measurement points. Resource use data, for costing, were collected using the Trimbos Institute and Institute of Medical Technology Assessment Questionnaire for Costs Associated with Psychiatric Illness (TiC-P) [15]. The TiC-P is the most widely used health service interview in the Netherlands. It consists of questions on the number of contacts by type of health care provider and questions on productivity losses. A health service questionnaire is a valid and reliable method for quantifying costs in trial-based economic evaluations in health care [16]. A cross-validation sample comparing TiC-P self-report to electronic patient files showed all data to be reliable, except for the number of reported sessions with a psychologist (data available upon request from first author). Not all patients had understood that they needed to incorporate the $16 \mathrm{VR}-\mathrm{CBT}$ sessions into their TiC-P self-report. This information was, therefore, $100 \%$ cross-checked using electronic patient files. The main cost driver was admission to psychiatric hospitals, so the number of days admitted to a psychiatric hospital was also $100 \%$ cross-checked against electronic patient files and corrected where needed.
Direct medical costs were calculated by multiplying health service units (eg, sessions, visits, and hospital days) with their standard economic cost price (see Multimedia Appendix 1). We also added the medication costs, consisting of antipsychotic and antidepressant medication. Corresponding costs were calculated as the cost price per standard daily dose, as reported in the Dutch Pharmaceutical Compass [17], multiplied by the number of prescription days, plus the pharmacist's dispensing costs of $€ 6$ per monthly prescription or $€ 12$ for a first-time prescription [18].

\section{Virtual Reality Costs}

For VR therapy hardware, software and training costs were calculated. Total yearly costs for one VR system was $€ 23,995$, according to CleVR BV, a company who builds VR sets. Yearly costs for training and supervision of the psychologists was $€ 13,400$. Per-patient costs per 16 VR-CBT treatment sessions was $€ 373.95$.

\section{Travel Costs}

Travel costs arose when participants had to make return trips for receiving health care at health services. Travel costs were computed as the average distance to a health service $(7 \mathrm{~km})$ multiplied by the costs per $\mathrm{km}(€ 0.21)$ [18].

\section{Productivity Costs}

Research assistants monitored changes in the participants' work status at baseline and at 3 and 6 months postbaseline using the TiC-P. Productivity losses in paid work were calculated according to the human capital approach [19], reflecting changes in the contractual number of hours worked per week and adjusting these for work-loss days arising from sick leave over the full period of 6 months using gender-specific hourly productivity costs [18]. Costs were originally expressed in Euros for the reference year 2014, but indexed to 2015 using the consumer price index as reported by Statistics Netherlands. In the reference year 2015, 1 Euro in the Netherlands equaled 1.235 US\$.

\section{Statistical Analysis}

\section{Imputation}

Following the CONSORT (Consolidated Standards of Reporting Trials) and CHEERS (Consolidated Health Economic Evaluation Reporting Standards) guidelines, all our analyses adhered to the intention-to-treat principle. To that end, missing values were imputed using multiply imputed chained equations (MICE) for nonparametric data with $\mathrm{M}$ of 100 bootstraps for each incomplete variable. Baseline variables predictive of effects (ie, QALYs and treatment response) were used for imputation, such as baseline data of the variable with missing values, treatment condition, ethnicity, education, sex, age, and safety behaviors at baseline. Safety behaviors, such as avoiding eye contact or escaping from social situations, were measured using the Safety Behaviour Questionnaire-Persecutory Delusions (SBQ-PD) [20]. Time spent with others showed a large difference at baseline despite randomization and was added as covariate in the CEA where time spent with others was used as the treatment response outcome of interest. 


\section{Main Analysis}

Both the CUA and CEA were conducted from the societal perspective. In each of these analyses, the incremental cost-effectiveness ratios (ICERs) were calculated as the between-group cost difference divided by the between-group effect difference. The ICER is interpreted as the additional costs per additional unit effect (ie, per additional treatment responder; per QALY gained). Cost and effect differences were obtained from seemingly unrelated regression equations of costs and effects, thus allowing for correlated residuals in the equations. The seemingly unrelated regression equations (SURE) models were bootstrapped 5000 times. In each bootstrap step, the mean cost differences and the mean outcome differences were computed and these were plotted on the cost-effectiveness plane. Finally, cost-effectiveness acceptability curves (CEACs) were graphed. CEACs inform decision makers about the likelihood that an intervention is deemed cost-effective, given a range of willingness-to-pay ceilings for gaining 1 QALY and gaining 1 treatment responder. All analyses were conducted in Stata, version 13.1 (StataCorp).

\section{Sensitivity Analyses}

The following sensitivity analyses were carried out. First, a sensitivity analysis was done including safety behavior at baseline as a covariate because despite randomization there was a significant difference at baseline, and it was found to be the main mediator in reducing paranoid ideation [10]. Second, a sensitivity analysis was done including psychiatric admission costs at baseline as a covariate because there was a large difference between groups at baseline. Third, a sensitivity analysis was done including both safety behavior at baseline and psychiatric admission costs at baseline as covariates in the model.

\section{Results}

\section{Overview}

After providing informed consent, 116 participants agreed to participate: $58(50.0 \%)$ in the control condition and $58(50.0 \%)$ in the experimental condition (see Figure 1).

Baseline characteristics of the sample can be found in Table 1. Results of costs and outcomes can be found in Table 2. A small group of participants was responsible for a large portion of the baseline costs, largely related to hospital admissions. The total days of psychiatric admissions were 233 days at baseline, 101 days posttreatment, and zero days at follow-up for the VR-CBT group. The total days of psychiatric admissions were 138 days at baseline, 20 days posttreatment, and 68 days at follow-up for the TAU group. 
Figure 1. Trial flow diagram. *Specification of participants lost to posttreatment: 6 declined further participation and 2 were lost due to clerical errors by therapist. $¥$ Specification of participants lost to follow-up: 9 declined further participation, 1 died of unrelated causes, and 2 were lost due to clerical errors by therapist. First published in Lancet Psychiatry (Pot-Kolder et al, 2018). VR-CBT: virtual reality-based cognitive behavioral therapy.

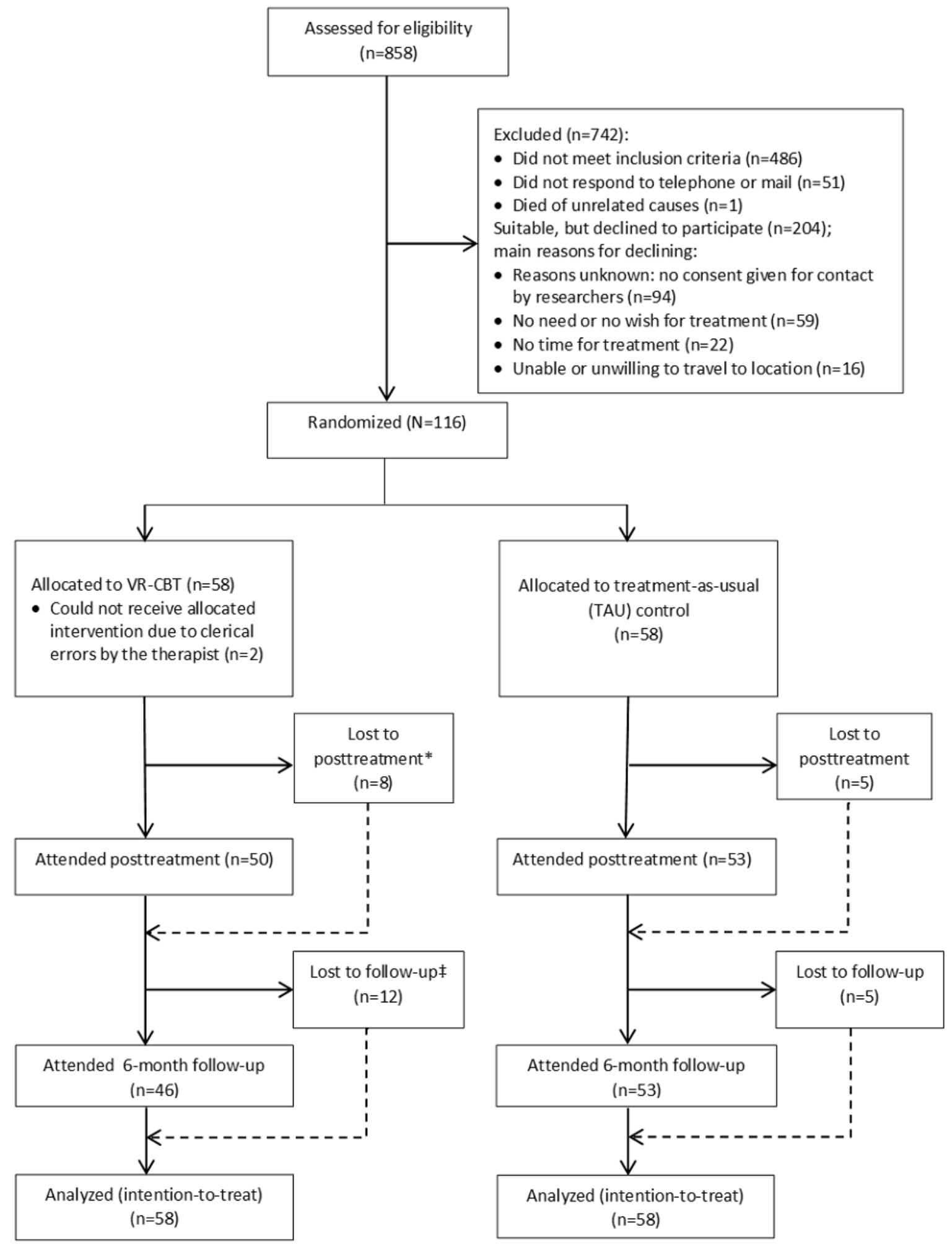


Table 1. Characteristics of the study sample at baseline.

\begin{tabular}{|c|c|c|}
\hline Characteristic & VR-CBT ${ }^{\mathrm{a}}(\mathrm{n}=58)$ & Treatment as usual (TAU) $(\mathrm{n}=58)$ \\
\hline Gender (male), n (\%) & $40(69)$ & $42(72)$ \\
\hline Age in years, mean (SD) & $36.5(9.8)$ & $39.5(10.0)$ \\
\hline Non-Dutch origin, $\mathrm{n}(\%)$ & $15(26)$ & $25(43)$ \\
\hline \multicolumn{3}{|l|}{ Education, $\mathbf{n}(\%)$} \\
\hline No education or primary & $16(28)$ & $16(28)$ \\
\hline Vocational & $18(31)$ & $24(41)$ \\
\hline Secondary & $9(16)$ & $9(16)$ \\
\hline Higher & $15(26)$ & $9(16)$ \\
\hline \multicolumn{3}{|l|}{ DSM-IV'diagnosis } \\
\hline Schizophrenia, n (\%) & $46(79)$ & $49(85)$ \\
\hline Schizoaffective disorder, $\mathrm{n}(\%)$ & $1(2)$ & $5(9)$ \\
\hline Delusional disorder, $\mathrm{n}(\%)$ & $1(2)$ & $0(0)$ \\
\hline Psychotic disorder (not otherwise specified), n (\%) & $10(17)$ & $4(7)$ \\
\hline Duration of illness in years, mean (SD) & $13.3(10.6)$ & $14.9(9.5)$ \\
\hline \multicolumn{3}{|l|}{ Medication use } \\
\hline Antipsychotics, n (\%) & $54(93)$ & $57(98)$ \\
\hline $\begin{array}{l}\text { Olanzapine equivalent of prescribed antipsychotic medication ( } \mathrm{mg} / \mathrm{day}) \text {, mean } \\
\text { (SD) }\end{array}$ & $10.5(6.8)$ & $11.0(8.3)$ \\
\hline Antidepressants, n (\%) & $15(26)$ & $17(29)$ \\
\hline Paid work, n (\%) & $8(14)$ & $5(9)$ \\
\hline Safety behaviors, mean (SD) & $28.8(14.2)$ & $21.1(16.0)$ \\
\hline
\end{tabular}

${ }^{\mathrm{a}}$ VR-CBT: virtual reality-based cognitive behavioral therapy.

${ }^{b}$ DSM-IV: Diagnostic and Statistical Manual of Mental Disorders, Fourth Edition.

Table 2. Average per-participant costs per 3-month period in Euros for the year 2015 and average outcomes by measurement and condition.

\begin{tabular}{|c|c|c|c|c|c|c|}
\hline \multirow[t]{2}{*}{ Costs and outcomes } & \multicolumn{2}{|l|}{ Baseline } & \multicolumn{2}{|c|}{ Posttreatment (3 months) } & \multicolumn{2}{|c|}{ Follow-up (6 months) } \\
\hline & VR-CBT ${ }^{\mathrm{a}}$ & $\mathrm{TAU}^{\mathrm{b}}$ & VR-CBT & TAU & VR-CBT & TAU \\
\hline \multicolumn{7}{|l|}{ Costs $(€)$, mean $(\mathrm{SD})$} \\
\hline Health care costs & $1918(5178)$ & $1396(3146)$ & $3031(3189)$ & $648(960)$ & $887(1160)$ & $1039(2640)$ \\
\hline Travel costs & $31(23)$ & $29(26)$ & $60(34)$ & $23(15)$ & $28(22)$ & $24(16)$ \\
\hline Productivity loss & $553(2730)$ & $224(1214)$ & 359 (1205) & 214 (1127) & $28(161)$ & $102(588)$ \\
\hline Total (societal) costs & $2502(6246)$ & $1649(3570)$ & $3076(3469)$ & $885(1589)$ & $943(1185)$ & $1165(2766)$ \\
\hline \multicolumn{7}{|l|}{ Outcomes, mean (SD) } \\
\hline GPTS $^{\mathrm{c}}$ paranoia (score) & $85(34)$ & $77(31)$ & $70(31)$ & $75(31)$ & $67(33)$ & $75(33)$ \\
\hline Time spent with others (proportion) & $0.416(0.256)$ & $0.364(0.266)$ & $0.404(0.226)$ & $0.323(0.266)$ & $0.419(0.209)$ & $0.340(0.273)$ \\
\hline Momentary anxiety $\left(\right.$ score $^{\mathrm{d}}$ ) & $2.986(1.120)$ & $3.259(1.484)$ & $2.586(1.089)$ & $3.221(1.495)$ & 2.645 (1.095) & $3.218(1.388)$ \\
\hline Momentary paranoia $\left(\right.$ score $^{\mathrm{d}}$ ) & $3.064(1.393)$ & $3.259(1.418)$ & $2.714(1.291)$ & $3.221(1.518)$ & $2.719(1.293)$ & $3.218(1.467)$ \\
\hline
\end{tabular}

${ }^{\mathrm{a}}$ VR-CBT: virtual reality-based cognitive behavioral therapy.

b TAU: treatment as usual.

'GPTS: Green's Paranoid Thoughts Scale.

${ }^{\mathrm{d}}$ Scores are on a 7-point Likert scale ranging from 1 (not at all) to 7 (very). 


\section{Incremental Effects}

\section{Time Spent With Others}

The treatment response rate regarding the time spent with others was 13 patients out of $58(22 \%)$ in the control group and 24 patients out of $58(41 \%)$ in the experimental group. The baseline-adjusted between-group difference between the response rates (ie, the incremental effect) was 0.23 , which was statistically significant $\left(\mathrm{SE}=0.076, \mathrm{t}_{113}=3.07,95 \%\right.$ CI $0.08-0.38$, $P=.003)$.

\section{Momentary Anxiety}

The treatment response rate with regard to momentary anxiety was 17 patients out of $58(29 \%)$ in the control group and 24 patients out of $58(41 \%)$ in the experimental group. The between-group difference between the treatment response rates (ie, incremental effect) was 0.12 , but this difference was not statistically significant $\left(\mathrm{SE}=0.089, \mathrm{t}_{114}=1.36,95 \% \mathrm{CI}-0.055\right.$ to $0.290, P=.18)$.

\section{Momentary Paranoia}

The treatment response rate in momentary GPTS paranoia was 11 patients out of $58(19 \%)$ in the control group and 28 patients out of $58(48 \%)$ in the experimental group. The between-group difference in the response rates was 0.29 and was statistically significant $\left(\mathrm{SE}=0.0841, \mathrm{t}_{114}=3.48,95 \%\right.$ CI 0.126-0.460, $P=.001$ ).

\section{Quality-Adjusted Life Years}

The SMD of GPTS paranoia was 0.523 , which was statistically significant $\left(\mathrm{SE}=0.120, \mathrm{t}_{114}=4.37,95 \% \mathrm{CI} 0.285-0.760, P<.001\right.$ ). Using Sanderson et al's conversion factor [14] of 0.1835 and taking into account a follow-up period of half a year, this became a QALY gain of $0.048(0.523 \times 0.1835 \times 0.5)$ favoring the VR-CBT condition and this was statistically significant $\left(\mathrm{SE}=0.011, \mathrm{t}_{114}=4.37,95 \%\right.$ CI $\left.0.026-0.069, P<.001\right)$.

\section{Incremental Costs}

\section{Incremental Health Care Costs}

As can be seen in Table 2, the average per-patient health care costs in the TAU group was $€ 1396$ at baseline, $€ 648$ at posttreatment, and $€ 1039$ at follow-up. The average per-patient health care costs in the VR-CBT group was $€ 1918$ at baseline, $€ 3031$ at posttreatment, and $€ 887$ at follow-up. This includes $€ 373.95$ per patient for VR-related costs included in the posttreatment costs.

The cumulative costs per patient between baseline and follow-up, including the costs of VR-CBT, were $€ 1686$ and $€ 3917$ in the TAU and VR-CBT conditions, respectively. The between-group difference was €2231 (€3917-€1686) and was statistically significant $\left(\mathrm{SE}=663, \mathrm{t}_{114}=3.36, P=.001\right)$ when not adjusted for the initial cost difference between the conditions at baseline. After adjustment for baseline costs, the incremental health care costs became slightly less at $€ 2170$ and retained statistical significance $\left(\mathrm{SE}=661, \mathrm{t}_{113}=3.28, P=.001\right)$.

\section{Incremental Costs Stemming From Productivity Losses}

A total of $11.2 \%(13 / 116)$ of the participants had paid work. The average costs stemming from productivity losses per person for the TAU group was $€ 224$ at baseline, $€ 214$ at posttreatment, and $€ 104$ at follow-up. The average costs stemming from productivity losses per person for the VR-CBT group was $€ 553$ at baseline, $€ 359$ posttreatment and $€ 28$ at follow-up. The cumulative costs per patient between baseline and follow-up were $€ 317$ and $€ 387$ in the TAU and VR-CBT conditions, respectively. The between-group difference was $€ 70$ (€387-€317) and was not statistically significant ( $\mathrm{SE}=274$, $\left.\mathrm{t}_{114}=-0.26, P=.80\right)$.

\section{Travel Costs}

The average costs stemming from travel per person for the TAU group was $€ 29$ at baseline, $€ 23$ at posttreatment, and $€ 24$ at follow-up. The average travel costs per person for the VR-CBT group was $€ 31$ at baseline, $€ 60$ at posttreatment, and $€ 28$ at follow-up. The cumulative travel costs per patient between baseline and follow-up were $€ 47$ and $€ 88$ in the TAU and VR-CBT conditions, respectively. The between-group difference was $€ 41$ (€88-€47) and was statistically significant ( $\mathrm{SE}=6$, $\left.\mathrm{t}_{114}=-6.73, P<.001\right)$.

\section{Incremental Costs From the Societal Perspective}

The cumulative societal costs per patient between baseline and follow-up were $€ 2050$ and $€ 4393$ in the TAU and VR-CBT conditions, respectively. The between-group difference was $€ 2343$ (€4293-€2050) and was statistically significant ( $\mathrm{SE}=747$, $\left.\mathrm{t}_{114}=-3.14, P=.002\right)$.

\section{Incremental Cost-Effectiveness Ratios From the Societal Perspective}

The mean incremental costs for a positive treatment responder was as follows:

1. Time spent with others: $€ 2343 / 0.23=€ 10,069$.

2. Momentary anxiety: $€ 2343 / 0.12=€ 19,525$.

3. Momentary paranoia: $€ 2343 / 0.29=€ 8079$.

4. The mean incremental cost per QALY: $€ 2343 / 0.048=€ 48,868$.

Figures 2 to 4 depict the distribution of the 5000 bootstrapped ICERs over the cost-effectiveness plane for each of the social participation measures. Figure 2 depicts time spent with others, the plane illustrates $99.70 \%$ of the ICERs fall in the northeast quadrant, indicating that more QALYs are gained for higher costs. Figure 3 depicts momentary anxiety, the plane illustrates 90.74\% of the ICERs fall in the northeast quadrant, indicating that more QALYs are gained for higher costs. Figure 4 depicts momentary paranoia, the plane illustrates $99.74 \%$ of the ICERs fall in the northeast quadrant, indicating that more QALYs are gained for higher costs.

Figure 5 depicts the distribution of the bootstrapped ICERs over the cost-effectiveness plane, with the vast majority of the ICERs in the northeast quadrant, indicating that more QALYs are gained but for higher costs, while $0.02 \%$ of the simulated ICERs fall in the southeast quadrant (ie, QALY gains for lower costs) for the VR-CBT group compared with the TAU group. 
Figure 2. Cost-effectiveness plane and willingness to pay (WTP) acceptability curve for time spent with others.
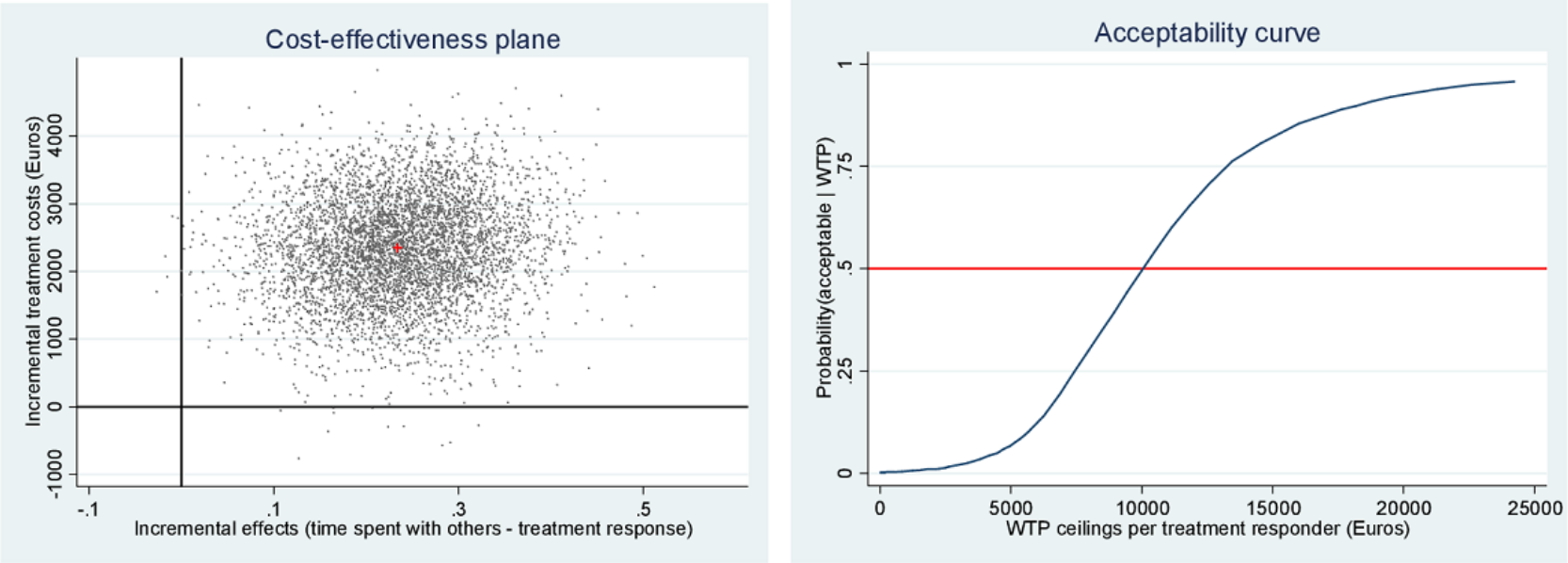

Figure 3. Cost-effectiveness plane and willingness to pay (WTP) acceptability curve for momentary anxiety.
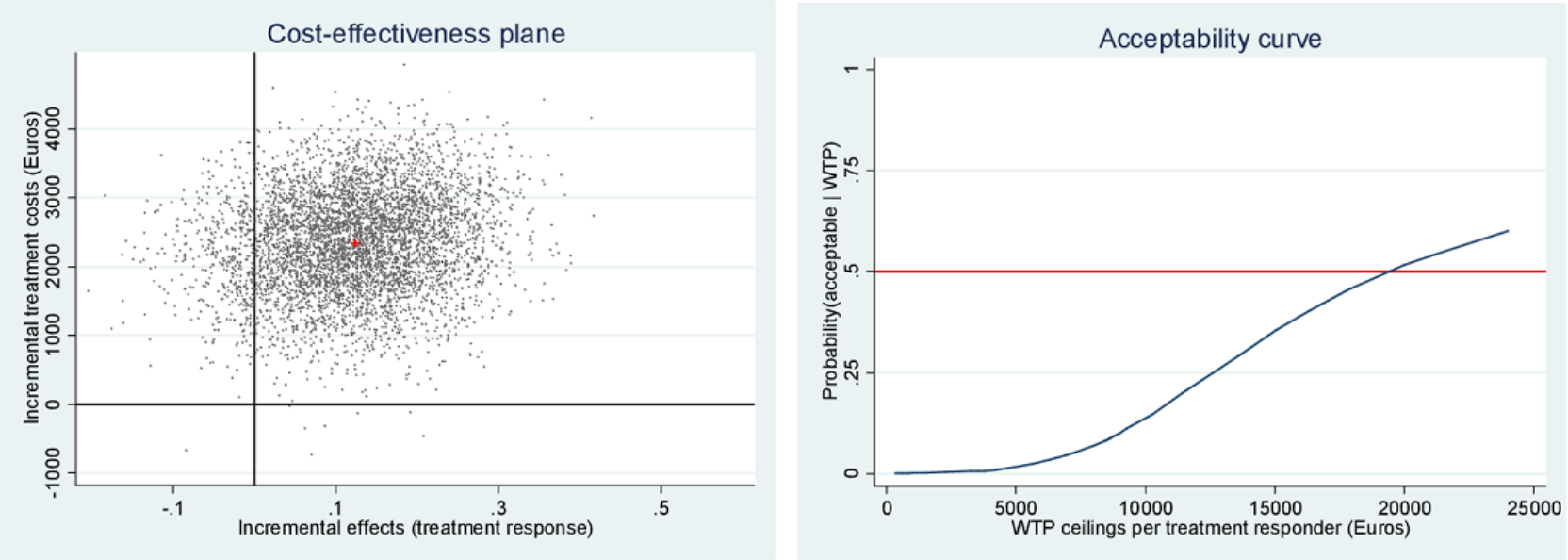

Figure 4. Cost-effectiveness plane and willingness to pay (WTP) acceptability curve for momentary paranoia.
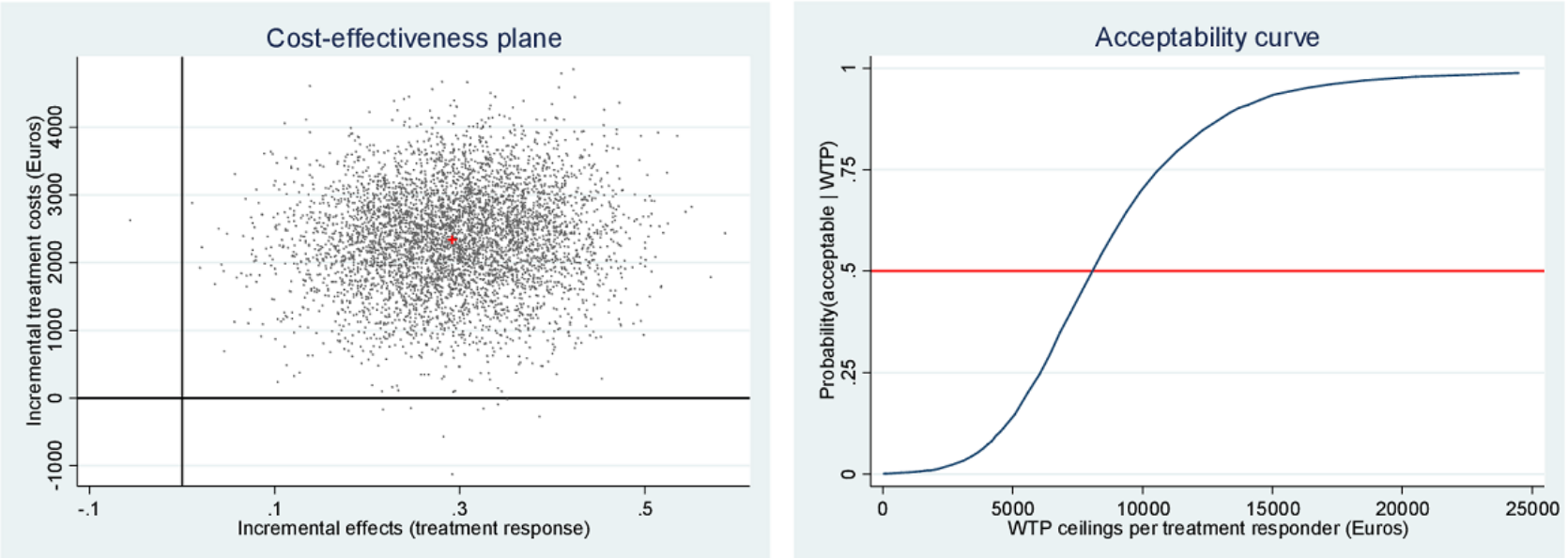
Figure 5. Cost-effectiveness plane and willingness to pay (WTP) acceptability curve for quality-adjusted life year (QALY) gain (costs per QALY gained) after 6 months.
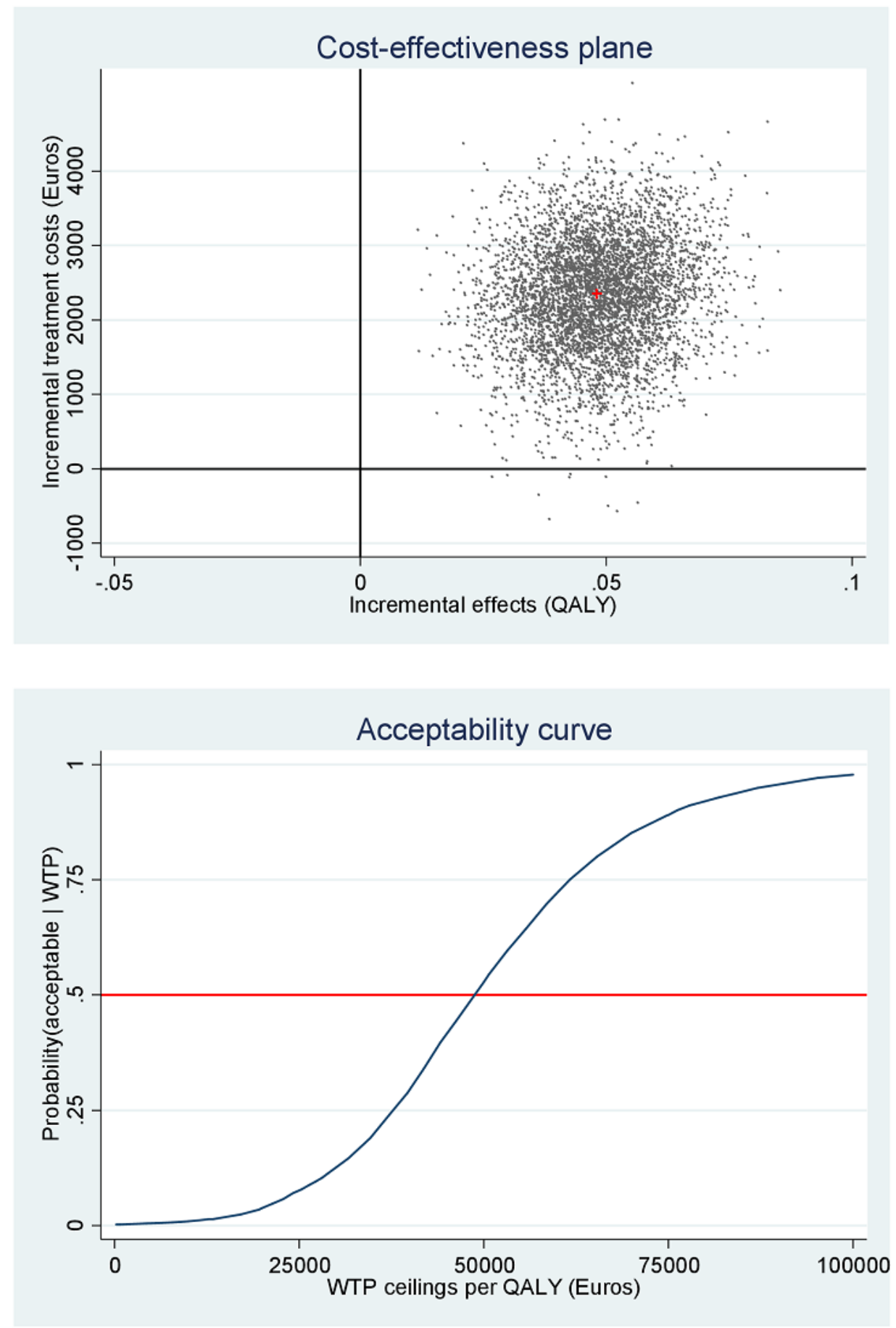

\section{Acceptability}

The mean incremental cost per QALY was $€ 48,868$. When looking at the acceptability curve in Figure 5, a higher probability that the VR-CBT intervention is deemed cost-effective can also be calculated. For an $80 \%$ certainty of cost-effectiveness, the incremental cost for gaining 1 QALY is $€ 66,161$, which falls well below the willingness-to-pay ceiling of $€ 80,000$ in the Netherlands for a severely disabling condition, such as schizophrenia characterized by paranoid delusions [21].

Looking at the three treatment responses, at $50 \%$ probability of being cost-effective, the costs are as mentioned: time spent with others, $€ 10,069$; momentary anxiety, $€ 19,525$; and momentary paranoia, €8079. Supposing that a decision maker needs an $80 \%$ certainty, time spent with others will have to be valued at $€ 14,293$ per treatment responder; momentary anxiety at $€ 50,000$; and momentary paranoia at $€ 11,342$.

\section{Sensitivity Analysis}

When including safety behavior at baseline as a covariate, the incremental costs per treatment responder on time spent with others became $€ 9136$; momentary anxiety became $€ 17,535$; and momentary paranoia became $€ 7219$. When including safety behavior at baseline as a covariate, the incremental costs per 
QALY gained became $€ 44,597$. Overall, incremental costs were somewhat lower when the baseline difference of safety behaviors was included in the analysis.

When including psychiatric admission costs at baseline as a covariate, the incremental costs per treatment responder on time spent with others became $€ 9729$; momentary anxiety became $€ 18,879$; and momentary paranoia became $€ 7750$. When including psychiatric admission at baseline as a covariate, the incremental costs per QALY gained became $€ 47,308$. Overall, incremental costs were somewhat lower when the baseline difference of psychiatric admission costs was included in the analysis.

When including both psychiatric admission costs at baseline and safety behavior at baseline as covariates, the incremental costs per treatment responder on time spent with others became $€ 8592$; momentary anxiety became $€ 16,597$; and momentary paranoia became $€ 6800$. When including both psychiatric admission costs and safety behavior at baseline as covariates, the incremental costs per QALY gained became $€ 42,030$. Overall, incremental costs were lower when the baseline differences of both safety behaviors and psychiatric admission costs were included in the analysis.

\section{Discussion}

\section{Principal Findings}

This study aimed to get an impression of short-term cost-effectiveness of VR-CBT for patients with paranoid delusions in comparison to TAU from a societal perspective. Data were collected 6 months after baseline at follow-up. Costs per treatment responder gained were estimated to be between $€ 8079$ and $€ 19,525$ for different aspects of social participation, with between $90.74 \%$ and $99.74 \%$ showing improvement. Cost per QALY gained at follow-up was estimated to be $€ 48,868$ with $99.98 \%$ showing improved QALYs. Sensitivity analyses showed costs to be lower when baseline differences in both safety behavior and psychiatric admission costs were included in the analysis. Costs per treatment responder gained were then estimated to be between $€ 6800$ and $€ 16,597$, with cost per QALY gained at $€ 42,030$.

\section{Results in Context}

How much a society values solidarity with people burdened by disease will determine if guidelines are translated to actual treatment of patients. While the VR-CBT treatment condition is more expensive than TAU only, that was to be expected, as the aim was to add to existing treatment. Results show that this addition improves social participation for people with a psychotic disorder suffering from paranoid ideation. We see this improvement for time spent with others, momentary paranoia, momentary anxiety, and paranoid ideation, via the GPTS.

Engaging in psychological therapy is challenging for many patients suffering from paranoid ideation and treatment results vary. There are several aspects that favor VR treatment.
Person-specific behavioral exposure is an important part of increasing treatment effect [22], which is exactly what the interactive VR social environments offer. Patients themselves also prefer VR over in vivo exposure treatment [23] and VR improves treatment motivation for patients [24].

Interestingly, during the follow-up we see that the VR-CBT group resulted in decreased health care costs and decreased costs due to productivity loss compared to the TAU-only group. There were no psychiatric admission days at follow-up for the VR-CBT group. To determine whether this was a coincidence or a trend, a much longer follow-up period is needed. Short-term societal costs were between $€ 8079$ and $€ 19,525$ for a positive treatment response. A disability weight of zero represents no loss of health and a weight of 1 represents health loss equivalent to death [25]. In the Netherlands, the willingness to pay for gaining a QALY ranges between $€ 20,000$ and $€ 80,000$ but differs per disease [26]. For a severely disabling disease such as schizophrenia, which according to the Global Burden of Disease study 2010 has a disability weight of 0.76 , the willingness to pay is $€ 80,000[21,26]$. In this context, the VR-CBT treatment that has an ICER of $€ 48,868$ per QALY gained can be regarded as acceptable from the cost-effectiveness point of view.

\section{Limitations}

The study has several limitations. First, data were collected only 6 months postbaseline. Any longer-term effects and costs are unknown. There are indications that cost-effectiveness for treatment of psychotic symptoms improves with time [27] as health benefits continue. Second, minimal treatment response was set at a $20 \%$ symptom reduction. A $20 \%$ symptom reduction after just 8 weeks of therapy is clinically relevant in a patient group with an average duration of illness of 14 years with persistently high problematic isolation. Third, VR-CBT was compared to TAU only. The next step would be to compare VR-CBT directly to CBT, which is the current gold standard, as CBT without VR also results in additional costs to TAU. There are, however, also indications that VR-CBT could have positive results in fewer sessions compared to CBT [9]. Comparing VR-CBT directly to CBT also allows for the study of presumed benefits of VR therapy, such as better engagement and the ecological validity of VR on outcome effects. Such a study comparing VR-CBT and CBT on time to response and costs is currently ongoing (Netherlands Trial Register number NL7758). A final limitation was that QALYs were not measured directly. As the EQ-5D (European Quality of Life Five Dimension Scale) was not administered, QALYs were calculated using Sanderson et al's conversion factor [14]. Future research needs to include the EQ-5D for direct measurement.

\section{Conclusions}

This study found VR-CBT to be cost-effective in the short term from a societal perspective. However, the effect of additional VR-CBT sessions and long-term effects need to be determined while using direct measurement of QALYs. 


\section{Acknowledgments}

Funding was received from Fonds NutsOhra, Stichting tot Steun VCVGZ.

\section{Conflicts of Interest}

None declared.

\section{Multimedia Appendix 1}

Direct medical costs. Prices are from 2015 and are in Euros. $* 8$ (sessions) $\times 2.5$ (hours) $\times 2$ (therapists) $\times 112$ (Euros per contact-hour) / 8 (participants) = G560. WRAP: Wellness Recovery Action Plan.

[PNG File, $60 \mathrm{~KB}-$ Multimedia Appendix 1]

\section{References}

1. Jin H, Mosweu I. The societal cost of schizophrenia: A systematic review. Pharmacoeconomics 2017 Jan;35(1):25-42. [doi: 10.1007/s40273-016-0444-6] [Medline: 27557994]

2. Neil AL, Carr VJ, Mihalopoulos C, Mackinnon A, Morgan VA. Costs of psychosis in 2010: Findings from the second Australian National Survey of Psychosis. Aust N Z J Psychiatry 2014 Feb;48(2):169-182. [doi: 10.1177/0004867413500352] [Medline: 24097844]

3. Tajima-Pozo K, de Castro Oller MJ, Lewczuk A, Montañes-Rada F. Understanding the direct and indirect costs of patients with schizophrenia. F1000Res 2015;4:182 [FREE Full text] [doi: 10.12688/f1000research.6699.2] [Medline: 26339474]

4. Moutoussis M, Williams J, Dayan P, Bentall RP. Persecutory delusions and the conditioned avoidance paradigm: Towards an integration of the psychology and biology of paranoia. Cogn Neuropsychiatry 2007 Nov;12(6):495-510. [doi: 10.1080/13546800701566686] [Medline: 17978936 ]

5. Perkins R, Rinaldi M. Unemployment rates among patients with long-term mental health problems. Psychiatr Bull 2018 Jan 02;26(8):295-298. [doi: 10.1192/pb.26.8.295]

6. Rosenheck R, Leslie D, Keefe R, McEvoy J, Swartz M, Perkins D, CATIE Study Investigators Group. Barriers to employment for people with schizophrenia. Am J Psychiatry 2006 Mar;163(3):411-417. [doi: 10.1176/appi.ajp.163.3.411] [Medline: $\underline{16513861]}$

7. Sibitz I, Amering M, Unger A, Seyringer ME, Bachmann A, Schrank B, et al. The impact of the social network, stigma and empowerment on the quality of life in patients with schizophrenia. Eur Psychiatry 2011 Jan;26(1):28-33. [doi: 10.1016/j.eurpsy.2010.08.010] [Medline: 21036554]

8. Degnan A, Berry K, Sweet D, Abel K, Crossley N, Edge D. Social networks and symptomatic and functional outcomes in schizophrenia: A systematic review and meta-analysis. Soc Psychiatry Psychiatr Epidemiol 2018 Sep;53(9):873-888 [FREE Full text] [doi: 10.1007/s00127-018-1552-8] [Medline: 29951929]

9. Freeman D, Bradley J, Antley A, Bourke E, DeWeever N, Evans N, et al. Virtual reality in the treatment of persecutory delusions: Randomised controlled experimental study testing how to reduce delusional conviction. Br J Psychiatry 2016 Jul;209(1):62-67 [FREE Full text] [doi: 10.1192/bjp.bp.115.176438] [Medline: 27151071]

10. Pot-Kolder RMCA, Geraets CNW, Veling W, van Beilen M, Staring ABP, Gijsman HJ, et al. Virtual-reality-based cognitive behavioural therapy versus waiting list control for paranoid ideation and social avoidance in patients with psychotic disorders: A single-blind randomised controlled trial. Lancet Psychiatry 2018 Mar;5(3):217-226. [doi: 10.1016/S2215-0366(18)30053-1] [Medline: 29429948]

11. Valmaggia LR, Latif L, Kempton MJ, Rus-Calafell M. Virtual reality in the psychological treatment for mental health problems: An systematic review of recent evidence. Psychiatry Res 2016 Feb 28;236:189-195. [doi: 10.1016/j.psychres.2016.01.015] [Medline: 26795129]

12. Pot-Kolder R, Veling W, Geraets C, van der Gaag M. Effect of virtual reality exposure therapy on social participation in people with a psychotic disorder (VRETp): Study protocol for a randomized controlled trial. Trials 2016 Jan 13;17:25 [FREE Full text] [doi: 10.1186/s13063-015-1140-0] [Medline: 26762123]

13. Green CE, Freeman D, Kuipers E, Bebbington P, Fowler D, Dunn G, et al. Measuring ideas of persecution and social reference: The Green et al Paranoid Thought Scales (GPTS). Psychol Med 2008 Jan;38(1):101-111. [doi: 10.1017/S0033291707001638] [Medline: 17903336]

14. Sanderson K, Andrews G, Corry J, Lapsley H. Using the effect size to model change in preference values from descriptive health status. Qual Life Res 2004 Sep;13(7):1255-1264. [doi: 10.1023/B:QURE.0000037482.92757.82] [Medline: 15473504]

15. Bouwmans C, De Jong K, Timman R, Zijlstra-Vlasveld M, Van der Feltz-Cornelis C, Tan Swan S, et al. Feasibility, reliability and validity of a questionnaire on healthcare consumption and productivity loss in patients with a psychiatric disorder (TiC-P). BMC Health Serv Res 2013 Jun 15;13:217 [FREE Full text] [doi: 10.1186/1472-6963-13-217] [Medline: $\underline{23768141]}$ 
16. van den Brink M, van den Hout WB, Stiggelbout AM, Putter H, van de Velde CJ, Kievit J. Self-reports of health-care utilization: Diary or questionnaire? Int J Technol Assess Health Care 2005;21(3):298-304 . [doi: 10.1017/s0266462305050397] [Medline: $\underline{16110708]}$

17. Farmacotherapeutisch Kompas (Pharmaceutical Compass). URL: https://www.farmacotherapeutischkompas.nl/ [accessed 2016-10-01]

18. Swan Tan S, Bouwmans-Frijters CAM, Hakkaart-van Roijen L. Handleiding voor kostenonderzoek: Methoden en referentieprijzen voor economische evaluaties in de gezondheidszorg. TSG 2012 Oct 11;90(6):367-372. [doi: $10.1007 / \mathrm{s} 12508-012-0128-3]$

19. Rice DP, Cooper BS. The economic value of human life. Am J Public Health Nations Health 1967 Nov;57(11):1954-1966. [doi: 10.2105/ajph.57.11.1954] [Medline: 6069745]

20. Freeman D, Garety PA, Kuipers E. Persecutory delusions: Developing the understanding of belief maintenance and emotional distress. Psychol Med 2001 Oct;31(7):1293-1306. [doi: 10.1017/s003329170100455x] [Medline: 11681555$]$

21. Council for Public Health and Health Care (Raad voor de Volksgezondheid en Zorg). Sensible and Sustainable Care (Zinnige en Duurzame Zorg) [document in Dutch]. Zoetermeer, the Netherlands: Council for Public Health and Health Care; 2006 Jun 07. URL: https://www.raadrvs.nl/binaries/raadrvs/documenten/publicaties/2006/06/07/zinnige-en-duurzame-zorg/ Advies_-Zinnige_en_duurzame_zorg.pdf [accessed 2016-10-01]

22. Turner DT, van der Gaag M, Karyotaki E, Cuijpers P. Psychological interventions for psychosis: A meta-analysis of comparative outcome studies. Am J Psychiatry 2014 May;171(5):523-538. [doi: 10.1176/appi.ajp.2013.13081159] [Medline: 24525715]

23. Carl E, Stein AT, Levihn-Coon A, Pogue JR, Rothbaum B, Emmelkamp P, et al. Virtual reality exposure therapy for anxiety and related disorders: A meta-analysis of randomized controlled trials. J Anxiety Disord 2019 Jan;61:27-36. [doi: 10.1016/j.janxdis.2018.08.003] [Medline: 30287083]

24. Rus-Calafell M, Garety P, Sason E, Craig TJ, Valmaggia LR. Virtual reality in the assessment and treatment of psychosis: A systematic review of its utility, acceptability and effectiveness. Psychol Med 2018 Feb;48(3):362-391. [doi: 10.1017/S0033291717001945] [Medline: 28735593]

25. Murray CJ, Vos T, Lozano R, Naghavi M, Flaxman AD, Michaud C, et al. Disability-adjusted life years (DALYs) for 291 diseases and injuries in 21 regions, 1990-2010: A systematic analysis for the Global Burden of Disease Study 2010. Lancet 2012 Dec;380(9859):2197-2223. [doi: 10.1016/S0140-6736(12)61689-4]

26. Zwaap J, Knies S, van der Meijden C, Staal P, van der Heiden L. Cost-Effectiveness in Practice (Kosteneffectiviteit in de Praktijk) [document in Dutch]. Diemen, the Netherlands: Dutch Healthcare Institute (Zorginstituut Nederland); 2015 Jun 26. URL: https://www.zorginstituutnederland.nl/binaries/zinl/documenten/rapport/2015/06/26/ kosteneffectiviteit-in-de-praktijk/Kosteneffectiviteit+in+de+praktijk.pdf [accessed 2016-10-01]

27. Ising HK, Lokkerbol J, Rietdijk J, Dragt S, Klaassen RM, Kraan T, et al. Four-year cost-effectiveness of cognitive behavior therapy for preventing first-episode psychosis: The Dutch Early Detection Intervention Evaluation (EDIE-NL) trial. Schizophr Bull 2017 Mar 01;43(2):365-374 [FREE Full text] [doi: 10.1093/schbul/sbw084] [Medline: 27306315]

\section{Abbreviations}

3DOF: 3 degrees of freedom

CBT: cognitive behavioral therapy

CEA: cost-effectiveness analysis

CEAC: cost-effectiveness acceptability curve

CHEERS: Consolidated Health Economic Evaluation Reporting Standards

CONSORT: Consolidated Standards of Reporting Trials

CUA: cost-utility analysis

DSM-IV: Diagnostic and Statistical Manual of Mental Disorders, Fourth Edition

EQ-5D: European Quality of Life Five Dimension Scale

ESM: ecological sampling method

GPTS: Green's Paranoid Thoughts Scale

ICER: incremental cost-effectiveness ratio

ISRCTN: International Standard Randomised Controlled Trial Number

MICE: multiply imputed chained equations

QALY: quality-adjusted life year

SBQ-PD: Safety Behaviour Questionnaire-Persecutory Delusions

SMD: standard mean difference

SURE: seemingly unrelated regression equations

TAU: treatment as usual

TiC-P: Trimbos Institute and Institute of Medical Technology Assessment Questionnaire for Costs Associated with Psychiatric Illness 
VR: virtual reality

VR-CBT: virtual reality-based cognitive behavioral therapy

VU University: Vrije Universiteit

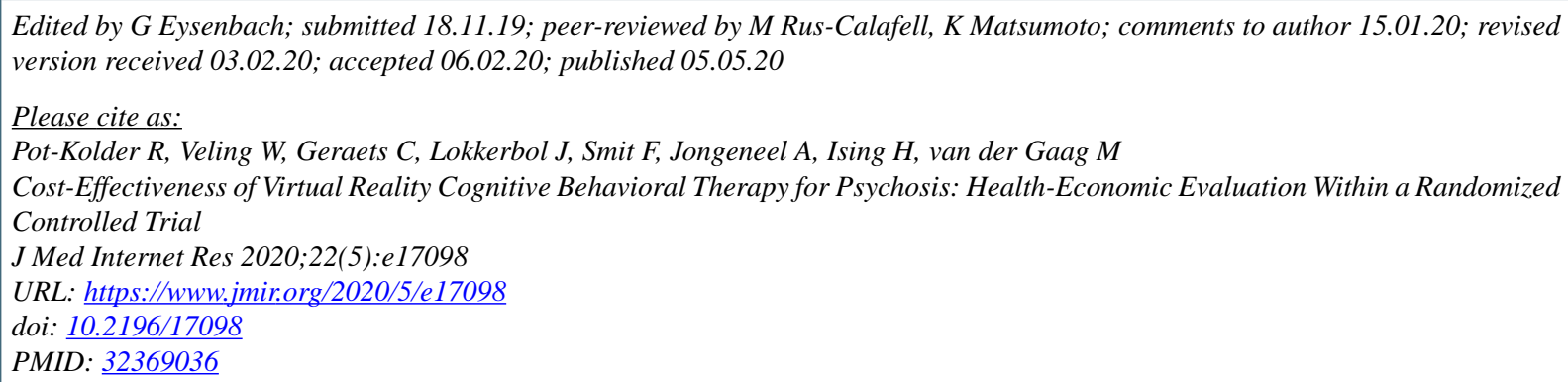

CRoos Pot-Kolder, Wim Veling, Chris Geraets, Joran Lokkerbol, Filip Smit, Alyssa Jongeneel, Helga Ising, Mark van der Gaag. Originally published in the Journal of Medical Internet Research (http://www.jmir.org), 05.05.2020. This is an open-access article distributed under the terms of the Creative Commons Attribution License (https://creativecommons.org/licenses/by/4.0/), which permits unrestricted use, distribution, and reproduction in any medium, provided the original work, first published in the Journal of Medical Internet Research, is properly cited. The complete bibliographic information, a link to the original publication on http://www.jmir.org/, as well as this copyright and license information must be included. 